$\underline{\xi}=-1$

\title{
A Novel Retinal Recognition System for Pathological Retina To Enhance Security
}

\author{
B.M.S.Rani ${ }^{*}$, A.Jhansi Rani ${ }^{2}$ \\ ${ }^{I}$ Research Scholar ANU, Nambur, Guntur (dst), AP, India. \\ ${ }^{2}$ Professor, V.R.Siddhartha engineering college, Vijayawada, AP, India. \\ *Corresponding Author E-mail: ${ }^{1}$ ranibms@gmail.com
}

\begin{abstract}
Biometric acknowledgment gives a characteristic and solid answer for the issue of individual distinguishing proof. One of the biometric ID framework utilized with high exactness is the retinal verification as a result of its many-sided quality in spoofing. However in these frameworks, acknowledgment rate is enormously influenced by the vasculature multifaceted nature of retinal images. This vascular example turns out to be exceptionally perplexing in sick retinal pictures because of the nearness of obsessive signs. In this manner, we require a computerized productive calculation which can evacuate these abnormalities before matching and decision making. The proposed novel hybrid clustering algorithm Adaptive Weighted Neighbour (AWN) Classification Algorithm classifies the input retinal image based on the features extracted and match the features with the trained features. The proposed feature vector consists of blood vessel structure, bifurcation points, bifurcation angles and vessel width. Results from the AWN are compared with state of the art classifier. It enables high security, good performance and greater accuracy. Also it provides better FAR, FRR and decreases the error rate.
\end{abstract}

Keywords: Biometric system, Blood vessels, identification, Retinal images, identification methods.

\section{Introduction}

Biometric acknowledgment gives a characteristic and solid answer for the issue of individual recognizable proof [1]. A biometric framework measures at least one physical or behavioral attributes (characteristics) to decide or check the individual personality by utilizing for instance, unique mark, palm print, confront, iris, retina, ear, voice, signature, walk, hand vein, smell, or the DNA data of an individual [2]. Such applications have been demonstrated as secure and have been utilized as a part of managing an account, welfare payment and security [3]. Biometrics have been proposed as a substitution for passwords, conquering some inalienable blemishes in secret key innovation [4]. Trusting totally in biometric innovation can prompt issues of its own, however [5].

One of the biometric identification system used with high accuracy is the retinal check in light of its many-sided quality in spoofing [6]. Computerized retinal pictures are utilized as a part of programmed ID frameworks [7]. They are procured utilizing computerized fundus camera comprising of low power magnifying instrument and utilized by eye experts for treatment of retinal infections [8]. The utilization of retinal acknowledgment frameworks are suited for high security military and Government territories because of their solidness and unwavering quality [9]. Primary concentrate was on the precise extraction of veins [10]. Various techniques have been proposed for vein recognition and division for PC helped symptomatic framework to identify retinal illnesses, however their utilization for retinal recognizable proof is extremely uncommon [11]. The structure of the veins of the retina is exceptionally intricate [12].

Each eye has its own particular absolutely special example of veins (even indistinguishable twins have unmistakable examples)
[13]. Albeit retinal examples might be changed if there should be an occurrence of sicknesses, for example, glaucoma, diabetes, and auto insusceptible proficiency disorder, the retina remains normally stable over a man's lifetime [14]. The execution of retina based biometric frameworks is considered as the best in term of precision [15]. In addition, human retina has not yet been fashioned and the retina of dead people rots too quickly to ever be utilized to swindle a retinal sweep [16]. Along these lines, with the exception of the way that retina check is frequently thought to be badly arranged and meddling so it is hard to increase general acknowledgment by the end clients, the retina is one of the body parts that fulfills splendidly all the attractive issues for a biometric framework [17].

Historic point based strategies for retinal enrollment utilizing vessel bifurcations and hybrid focuses as highlight focuses [18]. Another retinal enrollment strategy in view of area of optic circle is executed for retina confirmation [19]. A cross connection coefficient based retinal ID was finished. They initially enlisted the information picture and after that coordinating is finished by associating the vascular example. A vascular bifurcation and traverse point based framework is additionally actualized in the current techniques for retinal verification [20].

\section{Related Work}

Seyed Mehdi Lajevardi et al. [21] have proposed a programmed retina check structure in light of the biometric diagram coordinating (BGM) calculation.

The retinal vasculature was removed utilizing a group of coordinated channels in the recurrence area and morphological administrators. At that point, retinal layouts are characterized as formal spatial diagrams got from the retinal vasculature. The BGM calcu- 
lation, an uproarious chart coordinating calculation, strong to interpretation, nonlinear mutilation, and little revolutions, was utilized to look at retinal formats. The BGM calculation utilizes chart topology to characterize three separation measures between a couple of diagrams, two of which are new. A help vector machine (SVM) classifier was utilized to recognize bona fide and fraud examinations. Utilizing single and in addition numerous diagram measures, the classifier accomplished finish partition on a preparation set of pictures from the database (60\% of the information), breaking even with the best in class for retina check. Since the accessible informational collection was little, part thickness estimation $(\mathrm{KDE})$ of the certifiable and faker score appropriations of the preparation set are utilized to quantify execution of the BGM calculation.

Fabiola M. Villalobos-Castaldi and Ernesto Suaste-Gómez [22] have proposed a novel pupillary based confirmation framework, alongside the early personality verification results and examination, in view of the spatio-transient highlights processed from the unconstrained pupillary motions. The creators exhibit that this biometric attribute has the ability to give enough discriminative data to validate the character of a subject. Another strategy to figure the spatio-worldly biometric layout accounts of the understudy region changes, in a video-oculography grouping under steady luminance level. There is no confirmation that different endeavors were made, tending to this approach to recognize people in light of the spatio-fleeting portrayals, figured from the ordinary widening compression conduct of the understudy. In this work, liveness will be recognized by utilizing the data got from the unconstrained pupillary wavering component

Ireneusz Grulkowski et al. [23] have proposed a novel cleared source optical lucidness tomography (SS-OCT) imaging gadget utilizing a vertical cavity surface-discharging laser (VCSEL) fit for imaging the full eye length and presented a strategy utilizing this gadget for noncontact visual biometry. To think about the estimations of intraocular separations utilizing the SS-OCT instrument with industrially accessible optical and ultrasound biometers. The execution was assessed the intersession reproducibility of estimations of intraocular separations utilizing SS-OCT.

Fabiola M. Villalobos Castaldi et al. [24] have proposed another approach for retinal pictures highlights extraction and format coding. The utilization of the logarithmic winding testing lattice in examining and following the vascular system was the way to make this new approach straightforward, adaptable and solid. Examinations demonstrated that the utilized approach accomplished the lessening of information dimensionality and of the expected time to get the biometric code of the vascular system in a retinal picture. The performed tests exhibited that the confirmation framework has a normal precision of $95.0-98 \%$.

Hao et al. [25] have proposed a strategy utilizing shading histogram as the attribute of retina biometric. The shading histogram has demonstrated the component of distortion invariant. The upgrade utilizing discrete wavelet change disintegration limited the distinction inside the people and expands the relationship between's the retinal pictures of a similar individual. The utilized technique gave an answer against the variety inside the people and lower false dismissal rate in the retina biometric.

\section{Motivation}

Various biometric attributes are produced and utilized for individual distinguishing proof. The key thought is to utilize extraordinary attributes for individual ID, for example, confront, iris, retina hand and palm geometry, signature, voice and so on. The basic properties of these attributes for solid acknowledgment are the varieties of these qualities over the human populace, uniqueness and their changelessness after some time. Human retina is the best trademark when we consider the above characteristics for a biometric acknowledgment framework. It gives many points of interest over different qualities, for example, veins design in retina are extraordinary, thinks about have uncovered this reality that they are remarkable even in indistinguishable twins. They at last make a decent separation among individuals. Besides, they can't be copied and don't change over the time. Attributable to these properties, retina based ID and acknowledgment framework is the most steady and exact biometric framework. A solid and effective retina acknowledgment framework is described by the exactness of the framework. However in these frameworks, acknowledgment rate is significantly influenced by the vasculature many-sided quality of retinal pictures. For the most part, sound retina has slick example of veins. Division of these veins isn't a testing errand, as they don't have any anomalies in their example and neither do they impact the acknowledgment rate. Be that as it may, this vascular example turns out to be exceptionally perplexing in sick retinal pictures because of the nearness of neurotic signs. This obsessive sign show up as gaps and bundles in the retinal vascular guide. Hence, we require a robotized effective calculation which can expel these variations from the norm before coordinating and basic leadership. Administered classifiers is one of the current arrangement utilized as a part of the previous decades to beat the above issues. Be that as it may, such classifiers give low exactness because of wasteful component extraction techniques. This rouses us to propose a cross breed classifier with a reasonable element extraction strategy which extricates more number of highlights to enhance the exactness of characterization.

\section{Proposed Methodology}

In this proposed technique, three algorithms are accomplished for vein ID and confirmation. The Proposed technique portrays the approach of Enhancement algorithm and Classification process is utilized to accomplish the differentiation of vasculature especially in thin vessels and low difference vessels and concentrates the more number of highlights to improve the precision of characterization. At that point the improvement algorithm, grouping of altered algorithm and twofold morphological recreation is utilized concentrate the highlights, for example, edge amongst bifurcation and width of Retina Vessels retina.

The fundamental modules are pre-handling, Feature Extraction, Classification, Blood vessel estimation coordinating and decide the veins edge (bifurcation focuses) found in retinal veins. Underneath fig 1 . Demonstrates that engineering for proposed technique.

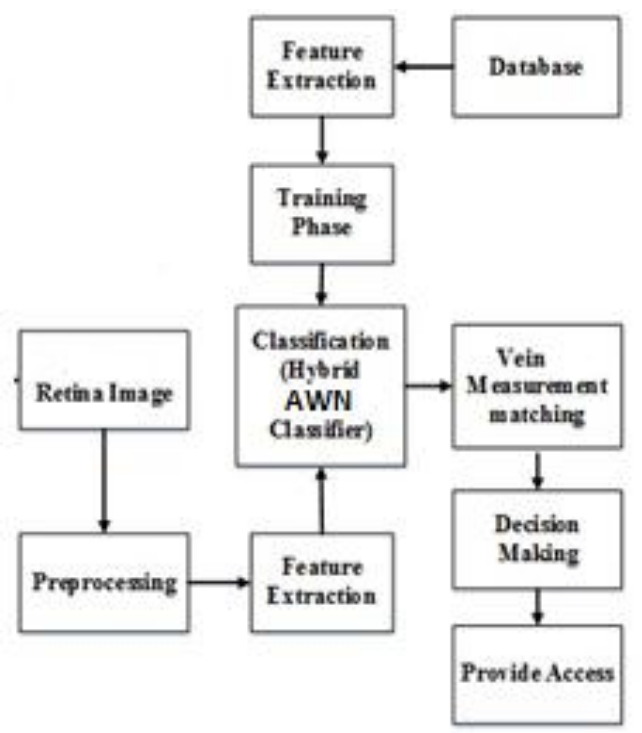

Fig. 1: Design for Proposed System

\subsection{Pre-processing Method}

Reflections, snapshot of patient, insufficient light, poor spotlight and horrendous course of action of positions drives low nature of 
picture so it debases the execution of the further examination process. In retinal biometric check right around $10 \%$ of the retinal pictures contains commotion and ancient rarities. Furthermore, retinal pictures ordinarily have a higher intricacy in the inside purpose of the image with lessened differentiation moving outward from the middle. For such pictures, a nearby differentiation upgrade method is utilized as a moment pre-handling step. At last it is required to influence a fundus to veil for each picture to energize the division of sores and anatomical structures in later stages. The pre-handling ventures in detail are depicted in the up and coming segments.

1. In this proposed work for pre-handling the info retinal picture is part into a non-covering squares.

2. At that point RGB parts are isolated from the first shading picture retina.

3. After that dim level transformation is performed utilizing histogram balance to enhance the difference and to upgrade the nature of picture retina.

4. At long last it utilize an expansive middle channel to expel the clamor from the picture.

Middle separating is one kind of smoothing procedure, as is straight Gaussian sifting. All smoothing algorithms are convincing at expelling clamors happened in smooth areas or smooth patches of any flag, yet incredulously influences the edges. Something else, all the while as lessening the clamor in a picture, it is basic to maintain the edges. Edges are requesting the need of the visual appearance of pictures, for instance. For little to relentless province of (Gaussian) clamor, the middle channel is plainly superior to anything Gaussian obscure at commotion expulsion and protecting edges for guaranteed, settled window measure. While, for spot clamor and salt and pepper commotion (indiscreet clamor), it is especially successful.

\section{A) Top-Hat Transformation}

In numerical morphology and electronic picture getting ready, topcap change is an operation that concentrates little parts and purposes of enthusiasm from given pictures. There exist two sorts of best cap change: The white best cap change which portrayed as the complexity between the data picture and its opening by some arranging segment; the dull best cap change which described dually as the qualification between the end and the data picture. Top cap changes are used for various picture taking care of endeavors, for instance, feature extraction, establishment evening out, picture improvement and others. Once the picture has been sifted, a preliminary area of veins is finished. The best cap change associated on the separated picture with a plate structure part with a size adequately far reaching to fill each one of the openings in veins. The best cap change is then performed by an end operation, which is described by the going with condition:

$$
T_{b}(f)=f \bullet b-f
$$

where,

$\mathrm{T}_{\mathrm{b}}(\mathrm{f})$ - transformation,

$(\bullet)$ - ending function,

b - organizing element and

f - filtered image.

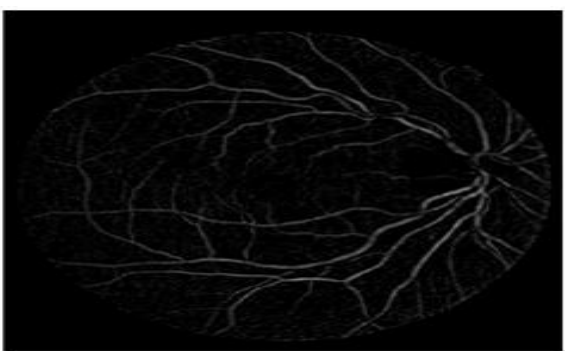

Fig. 2: Top Hat Transformation
The dimension, or width, of the parts that are expelled by the best top changes can be controlled by the decision of the dealing with portion $b$.The more significant masterminding part, the more prominent the sections evacuated. The best top changes is appeared in Fig. 2, the veins show up as clear expanded things while the foundation spreads to be dull.

Subtle elements removed by this method contain blood vessels as well as different points of interest from the foundation. In this image, we see an expansive distinction in contrast amongst thick and little blood vessels. Unmistakably thresholding techniques are not sufficiently appropriate to separate the vascular system. To take care of the issue of division, we utilize the morphology filtering.

\section{b) Morphological Filtering}

Double pictures may contain different blemishes. In particular, the double regions conveyed by direct thresholding are deformed by commotion and surface. Morphological picture getting ready looks for after the destinations of clearing these flaws by speaking to the structure and structure of the picture.

Morphological sifting operations can channel through any purposes of intrigue that are tinier in measure than the sorting out part from a picture, e.g., opening is separating the twofold picture at a scale portrayed by the degree of the arranging segment. Simply those portions of the picture that fit the sorting out segment are passed by the channel; more diminutive structures are blocked and stayed away from the yield picture. The traverse of the arranging part is most basic to discard uproarious inconspicuous components yet not to hurt objects of intrigue. We'll utilize morphological methodologies called "opening by propagation" and "opening and closing by changing" to "clean" up the picture. The templates from the retina are represented in the images figure 3 ( a) $\&$ ( b).

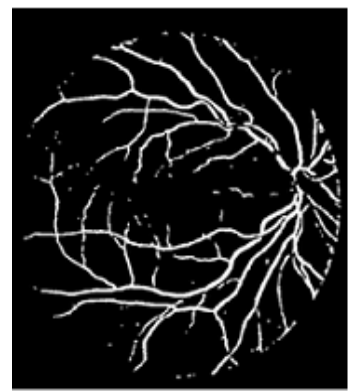

(A)

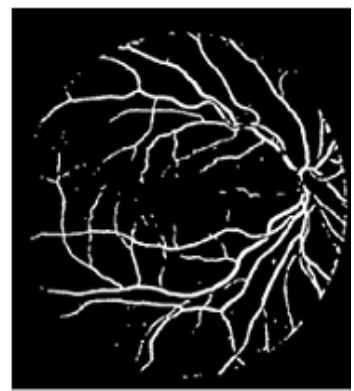

(B)
Fig. 3: (a) Opening by reconstruction, (b) Opening-closing by reconstruction

\subsection{Detection of Bifurcation Point}

The particulars extraction is most essential issue in extricate the bifurcation point. It is handled on skeleton picture by using a thought of intersection number $(\mathrm{CN})$. For an eight neighboring pixels to the inside pixels here $3 \times 3$ size pixel windows are utilized. In this window, if focal pixel is 1 and has precisely 3 one-esteem neighborhoods, at that point the focal pixel is a bifurcation point. For instance, if the focal pixel esteem is considered as 1and additionally it has just single adjacent values, at that point the focal pixels are specified as an edge finishing point (edge point where it closes) that constitute vein design. i.e. on the off chance that $\mathrm{CN}(\mathrm{P})==1$, it is an edge and if $\mathrm{CN}(\mathrm{P})==3$, then it is a bifurcation point. Each and every progression need to compute, so as to see either a pixel is a vessels bifurcation pointnor binded more than veins, put this window on the picture with the goal that the considered pixel is at the focal point of the window and discover the bifurcation point. In the event that the focal pixel does not lie on any vessel, it is not a bifurcation point. On the off chance that the middle pixel lie on the vessel then it is considered as a bifurcation point. 


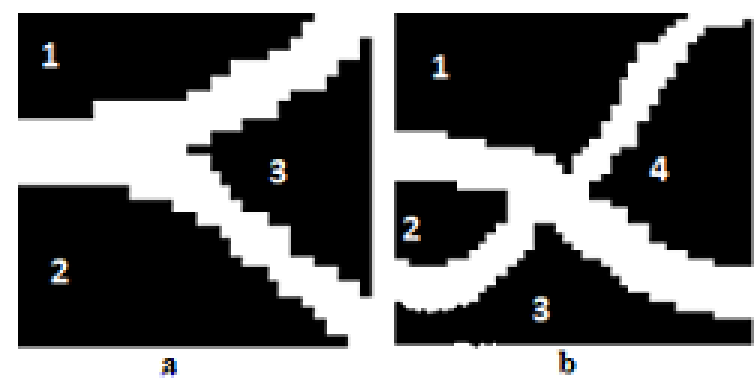

Fig. 4: (a). Bifurcation point, (b). Crossover point

\section{a. Detection of Angle}

The distinctive strides of Angle discovery algorithm for finding the edge between Two Lines of a Bifurcation Point is portrayed underneath.

1. Check any retinal twofold picture from its upper left corner to right-base corner. While crossing, a little inward window of any size is consider.

2. The double fundus picture is presently changed over into one pixel width skeleton shape. The white pixels are recognized when each pixel in the images are navigated. At that point check for the quantity of its encompassing neighboring pixels of white shading. (I) If the encompassing white pixels are not as much as a limit esteem, overlook those white pixels. The chose white pixels are then made intense by shading their encompassing four pixels as white (same shading as the picture) for a superior view.

(ii) If the encompassing pixels numbers are more than the limit (say 5-8), those white pixels are checked. Presently the checked white pixels are shaded dark (same as foundation) to get a solitary pixel skeleton of the picture

3. In the event that any bifurcation point happens, at that point the internal window transverse as clockwise. In the event that any progress emerges from white to dark, at that point spare the focuses and name the point as locale 1 , area 2 and 3 individually. Directly in every district, finds the beginning stages of the line as the bifurcation area is done itself by utilizing this algorithm. Likewise the same are sequenced for different locales.

4. At that point the slants of the districts are computed. Considering the slant of the primary line as $\mathrm{m} 1$ and the incline of the second line as $\mathrm{m} 2$. Calculates the angle between two lines by using formula as below

$$
\begin{gathered}
\tan \theta=\frac{m 1-m 2}{1+m 1 . m 2} \\
\theta=\tan ^{-1}\left(\frac{m 1-m 2}{1+m 1 . m 2}\right)
\end{gathered}
$$

Let us assume the starting points are at (u1, v1) and the final points are at (u2, v2).

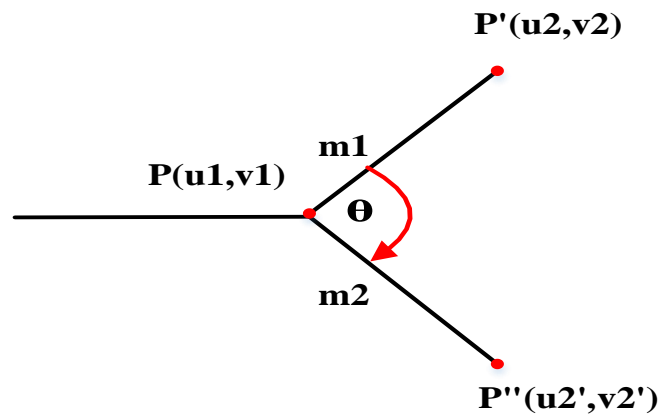

Fig. 5: Detection of angle between the bifurcation pointillustration

Assume that any bifurcation point from the fundusimage with starting points $(\mathrm{u} 1, \mathrm{v} 1)=(10,10)$ and end points of line $1(\mathrm{u} 2, \mathrm{v} 2)=$ $(12,30)$ and line $2\left(\mathrm{u} 2^{\prime}, \mathrm{v} 2^{\prime}\right)=(20,50)$
By considering $m 1=\frac{30-10}{12-10}$ and

$m 2=\frac{50-10}{20-10}$,

then the deatiled valueof $\mathrm{m} 1=10$ and $\mathrm{m} 2=4$.

Using this different step proposed algorithm, the angle between two lines of the bifurcation point is

$$
\theta=\tan ^{-1}(0.1463)
$$

Therefore, $\theta=8.3256$

\subsection{Measurement of Vessel Width}

The edge of the vessels is recognized by utilizing the picture and centerline. At that point the recognized picture will be mapped to discover the vessel width for a specific vessel centerline pixel position. For this reason single pixel is chosen from the centerline picture vessel, considering the veil at its inside. The cover is to locate the potential edge pixels in any side of that centerline pixel position. Consequently, the veil is connected to the edge pictures as it were. Rather than scanning for all the pixel positions inside the veil, width estimation technique compute the pixel position by moving by one up to the measure of the cover and in the meantime it pivot the position from 0 to 180 degrees. For expanding the pivot edge we utilize the measure of steps (contingent upon the veil estimate) under $180 /$ wavelength. Consequently, the strategy get to each cell in the cover utilizing this edge.

For every gray scale value obtained is analysed to check whether it is an edge pixel or not.

After finding out the edge of the pixel then find its opposition by changing the angle to 180 degree and extending the distance from minimum to the maximum size value of the mask

$x=x^{\prime}+r^{*} \cos \theta$ and $y=y^{\prime}+r^{*} \sin \theta$

where $\left(x^{\prime}, y^{\prime}\right)$ is the pixel position centre line vessel, $r=1,2, \ldots$ and $\theta=0, . ., 180$. For any pixel position, if the dark scale an incentive in the edge picture is 255 (white or edge pixel)at that point we finds out the pixel $(\mathrm{x} 2, \mathrm{y} 2)$ in the contrary edge(reflection of this pixel) considering $\theta=0+180$ and fluctuating $r$ this will be appeared in [17], [18].

Minimum Euclidian distance $=\sqrt{\left(x_{1}-x_{2}\right)^{2}-\left(y_{1}-y_{2}\right)^{2}}$

This enables us to find out the width for all blood vessels including the vessels with one pixel wide between the edge and the centre line itself.

Separation in the middle of point $\mathrm{c}$ and point $\mathrm{b}$ is figured relies upon (separation of Euclidean process)and the result variable is spared as $d$ is, the condition of The Euclidean separation (Dis) between two focuses $c$ and $b$ is:

For getting the base separation, $b$ guide (process toward the cover focus) is move to one side first pixel (right move for the veil) and figure the subtle elements of cover, if the result points of interest are not equivalent to esteem $d$ then it moves the cover to upper side or to the lower one pixel with a specific end goal to get d (this keep the focal point of the veilon the vein edge), at that point just the arrange esteems are characterized (the cover esteem constantly decided the directions).

Separation between point $\mathrm{c}$ and new point is registered, if the normal separation is lower than the predefined one then the new separation is spared as factor and new point organize spared as point $\mathrm{c}$. These strategy works over and over until the point that the separation wind up plainly bigger than the spared remove.

Table 1: Blood vessel types with its diameter

\begin{tabular}{|c|c|}
\hline Diameter & Blood vessel types \\
\hline Distance $\geq 11$ & Main vessel \\
\hline $4 \leq$ Distance $\leq 7$ & Secondary \\
\hline $2 \leq$ Distance $<3$ & Vascular \\
\hline
\end{tabular}




\subsection{Classification of Blood Vessels}

AWN classification method is used to the purpose of classify the clustered blood vessels with high accuracy. AWN classifies the input retinal image based on the features extracted and matches the features with the trained features. In our proposed method two level decision making is carried out because of the hybrid nature of our proposed classifier. In the initial level the concept of KNN classifier with hyper parameter of adaptive $\mathrm{k}$ size is implemented and classifies the nearest neighbours of the input image pixels. The clustered outputs are classified again using the concept of RF classifier by optimizing the number of trees which leads to high precious and accuracy in decision making. If the classifier verifies that the input retinal image is from the authorized user it provides an authentication and gives access to further processing.

In this algorithm two parameters expected to set up, first is the quantity of bunch $\mathrm{K}$, the other is $\mathrm{K}^{\prime}$ utilized amid every emphasis of k-closest neighbor. In this paper we pick arbitrary seed determination calculations since irregular is the straightforward. Generally it picks $\mathrm{k}$ focuses arbitrarily from the info set as the underlying focuses. The decision of $\mathrm{K}^{\prime}$ directly harmed the execution of order, if the parameter is too little, you can't discover enough report to legitimately arrange, else it would be discover more neighbors in the far off bunches, so the record would be appointed wrongly to more removed groups. We adjust a dynamic $\mathrm{K}^{\prime}$ change in every cycle, setting the closest neighbor parameter $K^{\prime}$ is the quantity of reports contained in the littlest class

\section{Algorithm for adaptive k' in AWN classifier}

Input: Training sample set TS, nearest neighbours W weights, the sample test set $\mathrm{S}$

Output: Category c

Initialization: Select $\mathrm{c} 1, \mathrm{c} 2, \ldots . . ., \mathrm{ck}$ in $\mathrm{C}^{\prime}$ as a group focus, each bunch is $\mathrm{C} 1, \mathrm{C} 2, \ldots . ., \mathrm{CW}$

Rehash

For each remain ci $\mathrm{C}^{\prime}$

Ascertain the similitude of ci and $c j, j=1,2 \ldots . \mathrm{k}$

On the off chance that $\operatorname{Sim}(\mathrm{ci}, \mathrm{cj})<\mathrm{Sim}(\mathrm{ci}, \mathrm{cn})$ at that point $\mathrm{Cn}=$ $\mathrm{Cn}\{\mathrm{ci}\}, \mathrm{n}=1,2, \ldots$.w djust the bunch focus

Until the point when every one of the pictures have their own particular bunch, which have an aggregate $\mathrm{K}$ groups

For each test pictures utilizing the versatile weighted neighbor algorithm to group informational collections

Repeat

For every S Do Begin

$\mathrm{W}^{\prime}=\mid$ the bunch containing minimal weights of images $\mid+1$

For $\mathrm{W}=1$ to $\mathrm{W}^{\prime} \mathrm{TS} 0$

$\mathrm{TSt}=$; in which TSt communicates the most comparable arrangement of pixels sets of test imaget

For every c Ci Do Begin

On the off chance that T exist z1 Cjand z2 Cimaking

$\operatorname{sim}(t, z 1)<\operatorname{sim}(t, z 2)$ at that point

Start

$\mathrm{TSt}=\mathrm{TSt}-\{\mathrm{z} 2\}$

$\mathrm{TSt}=\mathrm{TSt}\{\mathrm{z} 2\}$

End

End if

End

Until the point when the class of the considerable number of pixels are never again changed;

On the off chance that T and z1 TSt, z2 TSt and $|\mathrm{Cj}|>|\mathrm{Ci}|$ thent $\mathrm{Cj}$

End In the second step the least difficult random forest with random elements is framed by selecting at random, at every hub, a little gathering of information variables to part on. The steps are classified underneath

To build K trees

- Building every tree by
- Selecting, at random, at every hub a little arrangement of elements $(\mathrm{F})$ to part on (given $\mathrm{M}$ highlights). Basic estimations of $\mathrm{F}$ are:

- $1 . \mathrm{F}=1$

- 2. $\mathrm{F}=\log 2(\mathrm{M})+1$.

- For every hub separate the best of this subset (utilizing oob occasions).

Growing full length tree The proposed AWN calculation is a relationship between the adaptive k' and concept of random forest with optimized number of trees are prepared it called weighted neighbourhoods plans.

These two models worked from a preparation sets $\left\{\left(x_{i}, y_{i}\right)\right\}_{i=1}^{n}$ that make predictions $\hat{\boldsymbol{y}}$ for new points $\mathrm{x}^{\prime}$ by viewed at the point of "neighbourhood", formalized by a weight function $\mathrm{W}$,

$\hat{y}=\sum_{i=1}^{n} W\left(x_{i}, x^{\prime}\right) y_{i}$

Here, $\left\{\mathrm{W}\left(\mathrm{x}_{\mathrm{i}}, \mathrm{x}^{\prime}\right)\right\}$ is the non-negative weight of the $\mathrm{i}^{\prime}$ th training point relative to the new point $\mathrm{x}^{\prime}$.

For any particular $\mathrm{X}$ ' the weights must sum to one. Weight functions are given as follows:

In $\mathrm{k}-\mathrm{NN}, W\left(x_{i}, x^{\prime}\right)=\frac{1}{k}$ if 'xi' is one of the $\mathrm{k}$ points closest

to $\mathrm{x}^{\prime}$, and zero otherwise.

In $\mathrm{RF}, W\left(x_{i}, x^{\prime}\right)=\frac{1}{k^{\prime}}$ if ' $\mathrm{xi}$ ' is one of the $\mathrm{k}$ ' points in the same leaf as $x^{\prime}$, and zero otherwise.

Since a forest averages the predictions of a set of ' $m$ ' trees with individual weight functions $\mathrm{W}(\mathrm{j})$, its predictions are

$$
\begin{aligned}
& \hat{y}=\frac{1}{m} \sum_{j=1}^{m} \sum_{i=1}^{n} W\left(x_{i}, x^{\prime}\right) y_{i} \\
& \hat{y}=\sum_{i=1}^{n} \frac{1}{m}\left(\sum_{j=1}^{m} W\left(x_{i}, x^{\prime}\right) y_{i}\right)
\end{aligned}
$$

The neighbors of $x^{\prime}$ in this interpretation are the points $x(i)$ which fall in the same leaf as $x^{\prime}$ in at least one tree of the forest. In this way, the neighborhood of $\mathrm{x}^{\prime}$ depends in a complex way on the structure of the training set.

\section{Experimental Result}

The strategy was connected on the DRIVE database, which is worked for estimation of retinal acknowledgment frameworks. DRIVE database comprises aggregate of 40 shading fundus picture. Each picture is caught by a picture size of $565 \times 584$ with 24bits for every pixels. Which has been JPEG compacted. For the AWN classifiers, two sets are required; one for training and the other for testing, so the dataset is arbitrarily isolated into two arrangements of pictures, each contains 5 normal and 5 abnormal pictures. Arbitrary number of pixels are looked over the field of view (FOV) of each picture in the preparation set. The objectives for these preparation tests are accessible from the physically sectioned images. The testing set contains 10 pictures to test the execution of the classifier. For each pixel in every retinal picture in the dataset, a component vector is produced which contains four values - blood vessel structure ,bifurcation points, bifurcation angle, vessel width. In the first step the concept of knn is used with adaptive k' and Random forests work by generating a large number of decision trees, and averaging the results across trees. Thus one of the most obvious random forest parameters to optimize is the number of trees in the forest. As the number of trees in the forest increase, the accuracy improves, until it plateaus around 9 trees. Using around a four features in the dataset, which makes the hypothesize that forest should have a number of trees equal to twice the number of features. A grid search over tree depth, leaf size, and node size. 
The best parameters for this dataset and a four features was a maximum depth of 3 levels, minimum leaf size of 3, Optimizing these parameters yielded a $2-5 \%$ increase in accuracy over the default parameters.

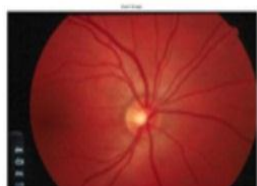

(a)

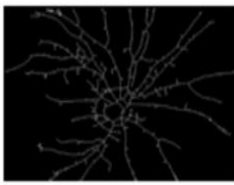

(d)

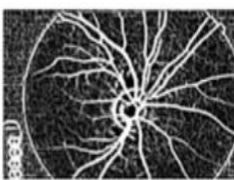

(b)

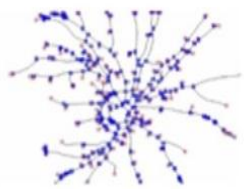

(e)

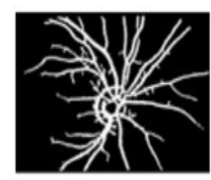

(c)
Fig. 6: (a)Input image of retina,(b) Pre-processed Image, (c) extracted image of retina d) Result of Thinning Process(e) Bifurcation point

The above fig 6(a) speaks to the contribution of unique retinal and fig 6(b) Speaks to picture after pre-handling strategy, Fig 6(c) Speaks to Feature Extracted picture, Fig 6(d) shows diminishing procedure of removed picture Fig.6(e) Shows the bifurcation focuses in numbers and limits. It likewise show the width of the vessel with specific vessel position.

In description manner of biometric framework there is an exclusive blunder that can impact the got execution. This mistake is known as The False Match Rate (FMR). It characterizes the likelihood that the framework erroneously coordinates the information Actually, it quantifies the percent of invalid info personalities which are erroneously allocated to the people. As this mistake is pretty much nothing, the framework is more productive.

By this paper we presume that characterization exactness of retinal pictures is perfect and productive by utilizing proposed strategies as contrast with existing techniques. In this paper, concentrate on two strategy for retinal picture bifurcation structure has been presented to be specific width coordinating and bifurcation point coordinating of veins. We delineates how highlight focuses are removed from retinal pictures and additionally its coordinating systems. Moreover includes are isolated utilizing details focuses which are organizes as width and point. Subsequent to applying the predefined informational index which gathers all extricated bifurcation and its directions. We gauged that this technique has better distinguishing proof accuracy and low blunder.

\subsection{Performance Analysis}

As we ascertain the inspecting rate of the proposed method techniques regarding the traverse time required to isolate the veins from the picture retina. We used info pictures from the DRIVE database to figure a normal preparing time for the proposed plan and similar pictures were utilized as contribution to the first programming based algorithm. The execution of our approach fundamentally in light of the picture estimate should be investigated and the I/O data transfer capacity. The qualities are organized in Table II and the correlation outline for approved and unapproved coordinating is graphically spoken.

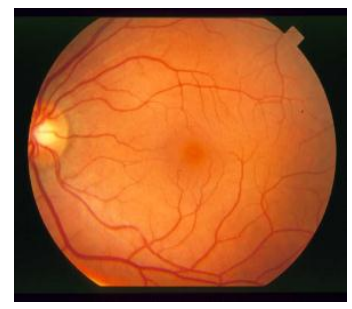

(a)

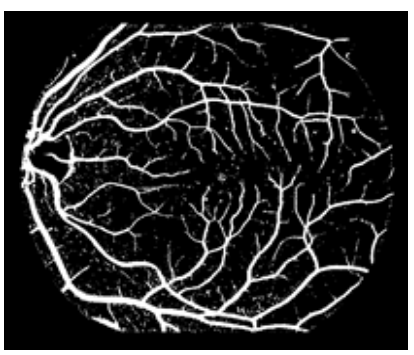

(b)

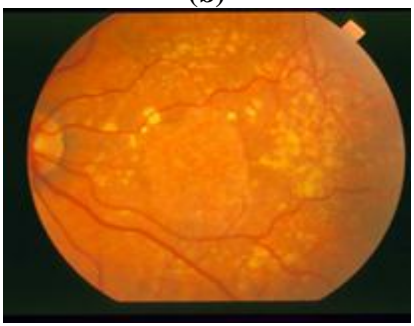

(c)

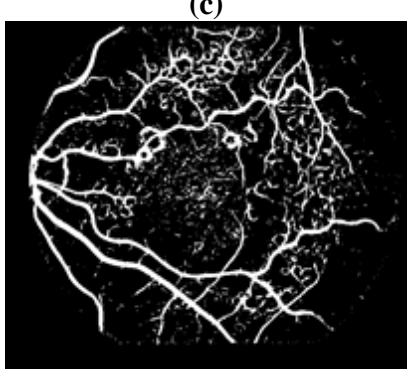

(d)

Fig. 7: (a) Normal Retina Fundus Image,(b) Abnormal retina fundus image, (c) \& (d) Blood Vessels

Table 2: AWN and SVM results

\begin{tabular}{|c|c|c|c|c|c|c|c|c|c|}
\hline |ethol & IIpe & Acculnary & FER & FAR & IRR & Sensitinity & Spentili & Ple & Aereall \\
\hline AIII & $\begin{array}{l}\text { Abromial } \\
\text { imgrgeg }\end{array}$ & & 73.256 & 65.0292 & 92.889 & 95.98 & 18.26 & 8.11 & 1.65 \\
\hline SIII & $\begin{array}{l}\text { Abromal } \\
\text { ingyege }\end{array}$ & & 82.038 & 81.3568 & 68.027 & 73.00 & 13.44 & 1958 & 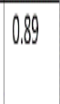 \\
\hline
\end{tabular}

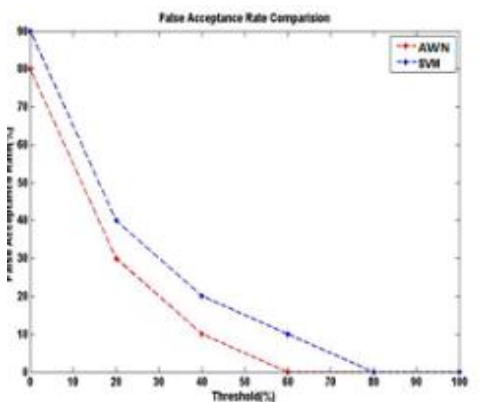

(a)

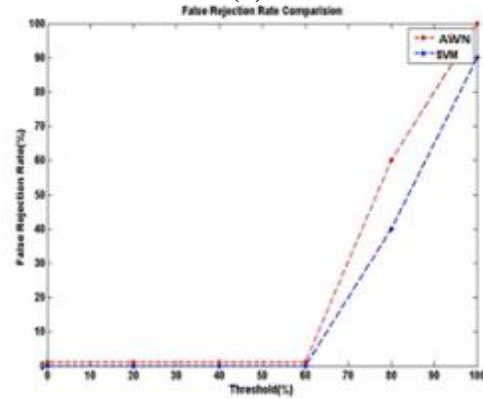

(b) 

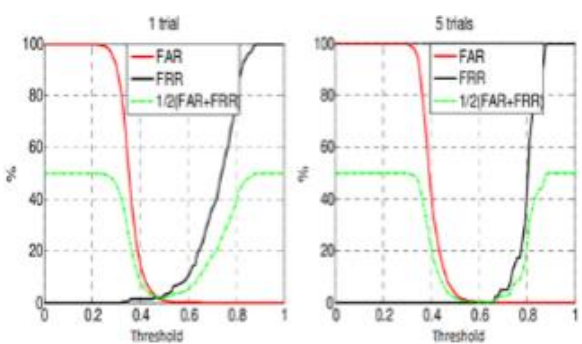

(c)

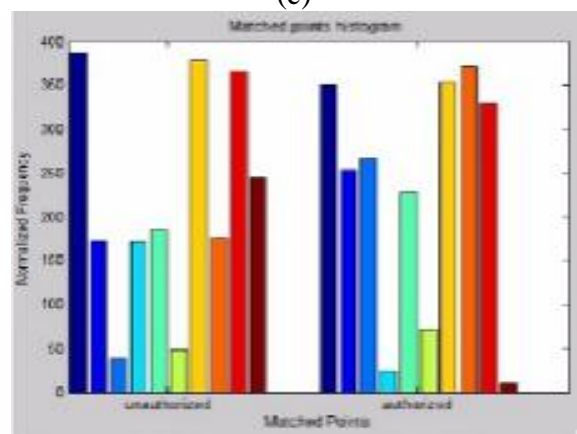

(d)

Fig. 8: (a) False Acceptance Rate Comparison with existing Classifier, (b)False Rejection Rate comparison between existing and AWN classifier,(c) Graphical Comparison of FAR vs. FRR,(d)Matched points Histogram

In the above figure 7.(a) is the normal retina fundus image used as input and fig.(b) is its blood vessel extracted image ,and figure 7.(c) is the abnormal retina fundus image used as input and fig.(d) is its blood vessel extracted image. The attainment of biometricsbased identification systems is also commonly depicted using FAR and FRR curves. The graph shows both the FAR and FRR curves is a characterization of the error between the FAR and the FRR as the threshold value is being changed. In Figure 8(a)\&(b), we displays the FAR and FRR curve for DRIVE dataset.

\section{Conclusion}

In this paper, we proposed a novel classification algorithm for abnormal retinal images in case of pathological signs to provide authentication based on the four feature vector consists of blood vessel structure ,bifurcation points ,bifurcation angles and vessel width. Experimental results show that the AWN algorithm offers $94.36 \%$ accuracy $95.98 \%$ sensitivity , and 18.26 specificity for abnormal images.

\section{References}

[1] Fatima, Joddat, Adeel M. Syed, and M. Usman Akram. "A secure personal identification system based on human retina." In Industrial Electronics and Applications (ISIEA), IEEE Symposium on, pp. 90-9, 2013.

[2] Hamid, Larry. "Biometric technology: not a password replacement, but a complement." Biometric Technology, no. 6, pp. 7-10, 2015.

[3] Modarresi, Morteza, and Iman Sheikh Oveisi. "A Contourlet Transform Based for Features Fusion in Retina and Iris Multimodal Biometric System." In Biometric Authentication, pp. 75-90. 2014.

[4] Patwari, Manjiri B., Ramesh R. Manza, Yogesh M. Rajput, Manoj Saswade, and Neha Deshpande. "Personal identification algorithm based on retinal blood vessels bifurcation." In Intelligent Computing Applications (ICICA), pp. 203-207, 2014.

[5] Ahmed, M. Islamuddin, M. Ashraful Amin, Bruce Poon, and Hong Yan. "Retina based biometric authentication using phase congruency."International Journal of Machine Learning and Cybernetics Vol.5, no. 6, pp. 933-945, 2014.

[6] Waheed, Zahra, M. Usman Akram, Amna Waheed, Muazzam A. Khan, Arslan Shaukat, and Mazhar Ishaq. "Person identification using vascular and non-vascular retinal features." Computers \& Electrical Engineering, 2016.
[7] Usher, David, Yasunari Tosa, and Marc Friedman. "Simultaneous Capture of Iris and Retina for Recognition." Encyclopedia of Biometrics, pp.1401-1407, 2015.

[8] Frucci, Maria, Daniel Riccio, Gabriella Sanniti di Baja, and Luca Serino. "Severe: Segmenting vessels in retina images." Pattern Recognition, 2015.

[9] Bartocha, Anna, Emil Saeed, Piotr Wachulec, and Khalid Saeed. "Retinal Feature Extraction with the Influence of Its Diseases on the Results." InApplied Computation and Security Systems, pp. 37 48, 2015.

[10] Koukounis, Dimitris, Christos Ttofis, Agathoklis Papadopoulos, and Theocharis Theocharides. "A high performance hardware architecture for portable, low-power retinal vessel segmentation." INTEGRATION, the VLSI journal Vol.47, no. 3, pp.377386, 2014.

[11] Drozd, Radek, Josef Hájek, and Martin Drahanský. "An algorithm for retina features extraction based on position of the blood vessel bifurcation." InBiometric Recognition, Springer Berlin Heidelberg, pp. 308-315, 2012.

[12] Unar, J. A., Woo Chaw Seng, and Almas Abbasi. "A review of biometric technology along with trends and prospects." Pattern recognition Vol.47, no. 8, pp.2673-2688, 2014.

[13] Seto, Yoichi. "Retina recognition." Encyclopedia of biometrics, pp.1321-1323, 2015

[14] Dehghani, Amin, Zeinab Ghassabi, Hamid Abrishami Moghddam, and Mohammad Shahram Moin. "Human recognition based on retinal images and using new similarity function." EURASIP Journal on Image and Video Processing, no. 1, pp.1-10, 2013.

[15] Panchal, Parth, Ronak Bhojani, and Tejendra Panchal. "An Algorithm for Retinal Feature Extraction Using Hybrid Approach." Procedia Computer Science Vol.79, pp. 61-68, 2016.

[16] Meng, Weizhi, Duncan S. Wong, Steven Furnell, and Jianying Zhou. "Surveying the development of biometric user authentication on mobile phones." Communications Surveys \& Tutorials, IEEE Vol.17, no. 3, pp.1268-1293, 2013.

[17] Ashokkumar, S., and K. K. Thyagharajan. "Retina biometric recognition in moving video stream using visible spectrum approach." In Green Computing, Communication and Conservation of Energy (ICGCE), pp. 180-187, 2013.

[18] Waheed, Zahra, Amna Waheed, and M. Usman Akram. "A robust non-vascular retina recognition system using structural features of retinal image." IEEE, pp. 101-105, 2016.

[19] Borah, Tripti Rani, Kandarpa Kumar Sarma, and Pran Hari Talukdar. "Retina recognition system using adaptive neuro fuzzy inference system." InComputer, Communication and Control (IC4), pp. 1-6, 2015.

[20] Chihaoui, Takwa, Rostom Kachouri, Hejer Jlassi, Mohamed Akil, and Kamel Hamrouni. "Human identification system based on the detection of optical Disc Ring in retinal images." In Image Processing Theory, Tools and Applications (IPTA), pp. 263-267, 2015.

[21] Lajevardi, Seyed Mehdi, Arathi Arakala, Stephen A. Davis, and Kathy J. Horadam. "Retina verification system based on biometric graph matching.", IEEE Transactions on Image Processing, Vol.22, no. 9, pp.3625-3635, 2013.

[22] Villalobos-Castaldi, Fabiola M., and Ernesto Suaste-Gómez. "A new spontaneous pupillary oscillation-based verification system." Expert Systems with Applications, Vol.40, no. 13, pp.5352 5362, 2013.

[23] Grulkowski, Ireneusz, Jonathan J. Liu, Jason Y. Zhang, Benjamin Potsaid, Vijaysekhar Jayaraman, Alex E. Cable, Jay S. Duker, and James G. Fujimoto. "Reproducibility of a long-range swept-source optical coherence tomography ocular biometry system and comparison with clinical biometers."Ophthalmology Vol.120, no. 11, pp. 2184-2190, 2013.

[24] Castaldi, Fabiola M. Villalobos, Edgardo M. Felipe-Riveron, and Ernesto Suaste Gómez. "A new retinal recognition system using a logarithmic spiral sampling grid." In Pattern Recognition, pp. 241$250,2014$.

[25] Hao, Hao, D. Krishna Kumar, Behzad Aliahmad, Che Azemin, Mohd Zulfaezal, and Ryo Kawasaki. "Using color histogram as the trait of retina biometric." In Biosignals and Biorobotics Conference (BRC), pp. 1-4, 2013. 